\title{
Model-Based Characterization of Atrial Fibrillation Episodes and its Clinical Association
}

\author{
Alba Martín-Yebra ${ }^{1}$, Mikael Henriksson ${ }^{1}$, Monika Butkuviené2 ${ }^{2}$, Vaidotas Marozas ${ }^{2}$, \\ Andrius Petrenas $^{2}$, Aleksei Savelev ${ }^{3}$, Pyotr G. Platonov ${ }^{4}$, Leif Sörnmo ${ }^{1}$ \\ ${ }^{1}$ Department of Biomedical Engineering, Lund University, Lund, Sweden \\ ${ }^{2}$ Biomedical Engineering Institute, Kaunas University of Technology, Kaunas, Lithuania \\ ${ }^{3}$ St. Petersburg State University, St. Petersburg, Russia \\ ${ }^{4}$ Arrhythmia Clinic, Skåne University Hospital, Lund, Sweden
}

\begin{abstract}
Studies investigating risk factors associated with atrial fibrillation $(A F)$ have mostly focused on AF presence and burden, disregarding the temporal distribution of $A F$ episodes although such information can be relevant. In the present study, the alternating, bivariate Hawkes model was used to characterize paroxysmal AF episode patterns. Two parameters: the intensity ratio $\mu$, describing the dominating rhythm (AF or non-AF) and the exponential decay $\beta_{1}$, providing information on clustering, were investigated in relation to AF burden and atrial echocardiographic measurements. Both $\mu$ and $\beta_{1}$ were weakly correlated with atrial volume ( $r=0.19$ and $r=0.34$, respectively), whereas $\mu$ was correlated with atrial strain $(r=-0.74, p \leq 0.1)$ and $A F$ burden $(r=0.68, p \leq 0.05)$. Weak correlation between $\beta_{1}$ and $A F$ burden was found ( $\left.r=0.29\right)$. Atrial structural remodeling is associated with changes in AF characteristics, often manifested as episodes of increasing duration, thus $\mu$ may reflect the degree of atrial electrical and structural remodeling. Moreover, clustering information $\left(\beta_{1}\right)$ is complementary information to AF burden, which may be useful for understanding arrhythmia progression and risk assessment of ischemic stroke.
\end{abstract}

\section{Introduction}

Atrial fibrillation (AF) is a heterogeneous disease. In paroxysmal AF, episode duration varies substantially, lasting from less than $30 \mathrm{~s}$ to 7 days. When investigating risk factors associated with $\mathrm{AF}$, minimal episode duration has been related to increased risk of thrombus formation [1]. The prognostic value of AF burden (the percentage of time the patient is in AF relative to the total monitoring period) for the risk of ischemic stroke has been established in several studies [1-3]. However, little is known about the role of temporal episode patterns in AF progression [4], although such information can be relevant for a better understanding of disease progression as well as for risk assessment of ischemic stroke.

A model-based approach to characterizing the pattern of AF episodes using an alternating, bivariate Hawkes model has been proposed in a recent study [5]. This model is a variant of the bivariate Hawkes point process model [6,7], in which a transition increases the likelihood of observing additional transitions in the near future (self-excitation property), accounting for clustered patterns. It provides a history-dependent modeling of both non-AF to AF and $\mathrm{AF}$ to non-AF transitions, being suitable for statistical inference due to the few parameters. Using the maximum likelihood (ML) method for parameter estimation, the goodness-of-fit analysis demonstrated that the proposed model fitted the data in the vast majority of recordings, supporting that a wide range patterns can be modeled. However, the clinical significance of the model parameters remains to be investigated.

The present study uses the above-mentioned model for characterizing AF episode patterns in long-term (1-7 days) ECG recordings from patients with paroxysmal AF. Thereafter, the clinical association between echocardiographic measurements, accounting for atrial structural remodeling, and model parameters, representing rhythm dominance ( $\mathrm{AF}$ or non-AF) and $\mathrm{AF}$ episode clustering, is investigated.

\section{Materials}

A database was acquired from patients with paroxysmal AF at the State University of St. Petersburg, Russia. The database consists of 37 three-lead ambulatory ECG recordings (sampling rate $257 \mathrm{~Hz}$ ) lasting from 1 to 7 days, summing up to a total of 160 days of data. Additional clinical information was collected at enrolment, including sex, age and anthropometric measures together with an echocardiogram test in a subgroup of 14 patients. For those patients, 
several echo-derived measurements as left atrial volume (LAV) and left atrial strain (LAS) were available (see Table 1). The study was approved by the local ethical review board.

\begin{tabular}{lc}
\hline \hline Variable & $\begin{array}{c}\text { Overall population } \\
(\mathrm{n}=14)\end{array}$ \\
\hline Age (years) & $62 \pm 4.9$ \\
Gender $($ males $)$ & $8(57.1 \%)$ \\
Height $(\mathrm{cm})$ & $171 \pm 8$ \\
Weight $(\mathrm{kg})$ & $82 \pm 12.5$ \\
Left atrial volume $(\mathrm{ml})$ & $60 \pm 16$ \\
Left atrial strain $(\%)$ & $27.8 \pm 12.3$ \\
\hline \hline
\end{tabular}

Table 1. Characteristics of patients. Data are presented as mean \pm standard deviation and as absolute frequencies (percentages).

\section{Methods}

The statistical framework used in this study to characterize AF episode patterns is described in detail in [5].

\subsection{Preprocessing}

After QRS detection using a wavelet-based approach [8], AF detection was performed involving information on ventricular rhythm, atrial rhythm, and f wave morphology [9]. In brief, four parameters serve as input:

1. RR irregularity, quantified by an entropy-based metric computed in an 8-beat sliding window [10].

2. P wave absence, quantified by computing the normalized ratio of the rectified signal in the PQ interval to that of the TQ interval.

3. f wave presence, quantified by the squared and summed error between different PR intervals.

4. noise level, quantified by the spectral entropy ratioweighted root mean square value of the extracted $f$ waves.

The latter three parameters are determined from an $\mathrm{f}$ wave signal, extracted using an echo state network [9]. The classifier requires no prior training. The database was annotated followed by manual review to find undetected AF episodes and to discard false alarms. The review was done by an expert on AF analysis, consulting other experts in doubtful cases.

\subsection{The alternating, bivariate Hawkes model}

The temporal pattern of AF episodes is modeled by two counting (point) processes: $N_{1}(t)$, accounting for transitions from non-AF to AF occurring at times $t_{1,1}, t_{1,2}, \ldots$, and $N_{2}(t)$ for transitions from AF to non-AF occurring
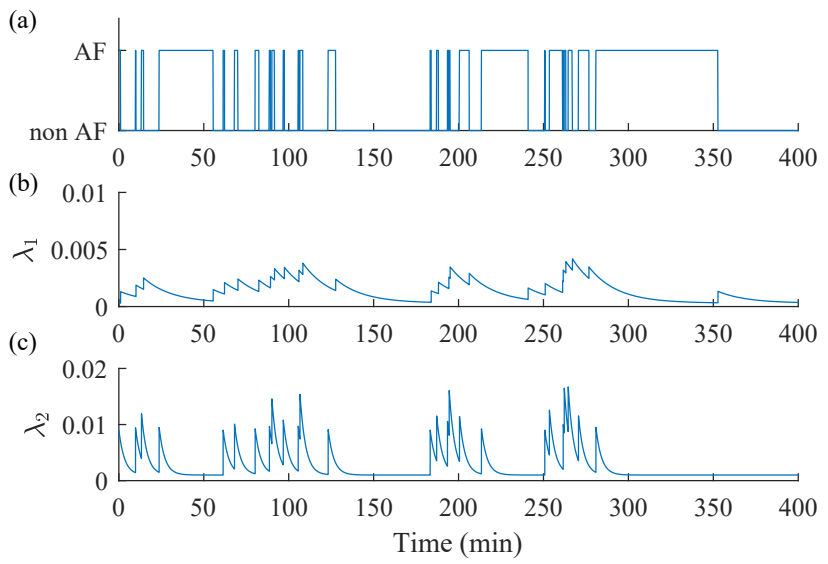

Figure 1. A realization of the alternating, bivariate Hawkes process: AF to non-AF sequence in (a); $\lambda_{1}(t)$ and $\lambda_{2}(t)$ are presented in panels (b) and (c), respectively.

at times $t_{2,1}, t_{2,2}, \ldots$. This bivariate point process is completely characterized by the two conditional intensity functions $\lambda_{1}(t)$ and $\lambda_{2}(t)$, defined by [11]:

$$
\lambda_{m}(t)=\lim _{\Delta t \rightarrow 0} \frac{\operatorname{Pr}\left(N_{m}(t+\Delta t)-N_{m}(t)=1 \mid \mathcal{H}_{t}\right)}{\Delta t},
$$

where the numerator is the conditional probability of a transition occurring in the interval $[t, t+\Delta t]$, and $\mathcal{H}_{t}$ is the history of the bivariate point process, i.e., the transition times $t_{1,1}, t_{2,1}, t_{1,2}, \ldots$ that have occurred up to but not including $t$.

Using the bivariate Hawkes model [6], these two conditional intensity functions take the following expression:

$$
\lambda_{m}(t)=\mu_{m}+\sum_{n=1}^{2} \sum_{\left\{k: t>t_{n, k}\right\}} \alpha_{m, n} e^{-\beta_{m, n}\left(t-t_{n, k}\right)},
$$

where $\mu_{m}>0, \alpha_{m, n} \geq 0, \beta_{m, n} \geq 0$ for $m, n=1,2$ and $t_{m, n}$ the occurrence times.

The principal characteristic of the Hawkes model is that each time a new point arrives, the conditional intensity $\lambda_{m}(t)$ grows by a factor of $\alpha_{m, m}$ ("self-excitation" property) or $\alpha_{m, n}$ ("cross-excitation" property), depending on the nature of the transition, exponentially decreasing (defined by the decay parameter $\beta_{m, n}$ ) towards the base intensity $\mu_{m}$. Since a transition increases the probability of getting other points immediately after, this is a model for clustered patterns (groups of episodes appearing close in time). A realization of the bivariate Hawkes point process with the associated $\lambda_{1}(t)$ and $\lambda_{2}(t)$ is illustrated in Fig. 1.

A disadvantage of the bivariate Hawkes model in its original form is that it does not impose alternation between transitions, i.e., a transition from SR to AF (onset of the episode) is not necessarily followed by a transition from AF to SR (end of the episode). To overcome this limitation, 
both $\lambda_{1}(t)$ and $\lambda_{2}(t)$ are multiplied by the corresponding "occurrence" function, defined as:

$$
\begin{aligned}
& o_{1}(t)= \begin{cases}1, & N_{1}(t)=N_{2}\left(t-d_{2}\right), \\
0, & \text { otherwise }\end{cases} \\
& o_{2}(t)= \begin{cases}1, & N_{2}(t) \neq N_{1}\left(t-d_{1}\right), \\
0, & \text { otherwise }\end{cases}
\end{aligned}
$$

where $d_{1}$ and $d_{2}$ are the minimum duration of AF and nonAF episodes, respectively, both set to $3 \mathrm{~s}$.

Finally, the conditional intensity functions describing the alternating, bivariate Hawkes process are given by

$$
\tilde{\lambda}_{m}(t)=\lambda_{m}(t) o_{m}(t), \quad m=1,2 .
$$

\subsection{Model parameters}

Assuming that $\beta_{1,1}=\beta_{1,2}=\beta_{1}$ and $\beta_{2,1}=\beta_{2,2}=\beta_{2}$ for simplicity, the model is defined by a total of eight parameters: $\mu_{1}, \alpha_{1,1}, \alpha_{1,2}$ and $\beta_{1}$ describing $\tilde{\lambda}_{1}(t)$ and $\mu_{2}, \alpha_{2,1}$, $\alpha_{2,2}$ and $\beta_{2}$ describing $\tilde{\lambda}_{2}(t)$.

The parameter $\beta_{1}$ may be related to episode clustering since a slow exponential decay (i.e., low value of $\beta_{1}$ ) increases the likelihood that an episode is followed by additional episodes. On the contrary, as $\beta_{1}$ increases, the decay of the intensity function towards the base intensity is faster, thus leading AF episodes appearing more spread in time. This effect is illustrated in Fig. 2 (a) and (b). On the other hand, due to the alternation between the two types of transitions (onsets and ends), the cross-excitation parameters $\alpha_{1,2}$ and $\alpha_{2,1}$ are usually much larger than the selfexcitation parameters $\alpha_{1,1}$ and $\alpha_{2,2}$. Finally, the base intensities $\mu_{1}$ and $\mu_{2}$ reflect the mean rates of non-AF to AF and AF to non-AF transitions, respectively, in the absence of self- and cross-excitation. We define the base intensity ratio as

$$
\mu=\frac{\mu_{1}}{\mu_{2}}
$$

which, depending on whether $\mu>1$ or $\mu<1$, indicates the dominance of AF or non-AF states, respectively (Fig. 2(b) and (c)).

The ML estimator was derived to find the model parameters from real data (we refer the reader to [5] for details). ML estimation is performed in ECG recordings with at least 10 episodes, i.e., 20 transitions.

\section{4. $\quad$ Results}

Two model parameters, $\beta_{1}$ and $\mu$, were associated with two echo measurements, LAV and LAS, reflecting mechanical atrial performance (Fig. 3). Unfortunately, this
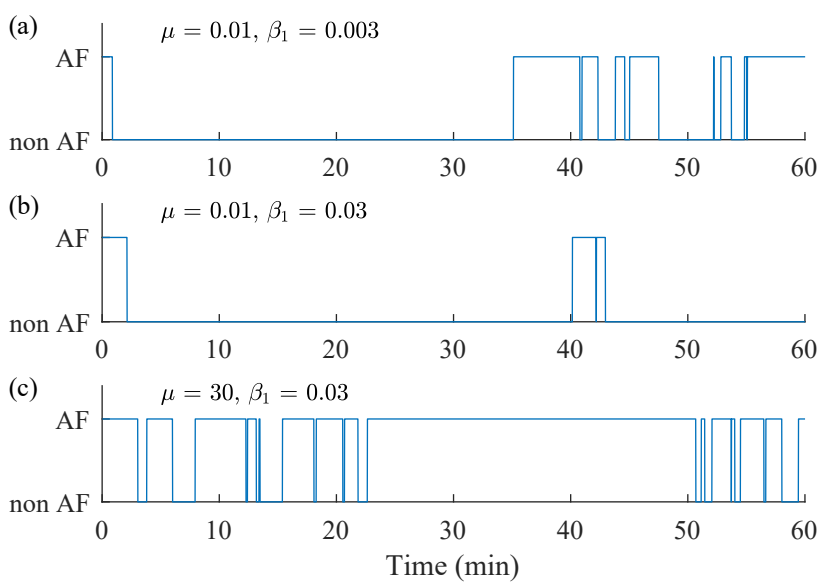

Figure 2. (a) Episode pattern dominated by non-AF and clustered episode pattern. (b) Episode pattern dominated by non-AF and increasing $\beta_{1}$ from 0.003 to 0.03 , leading to less clustering. (c) Episode pattern dominated by AF, increasing $\mu$ from 0.01 to 30 .

analysis was limited to a small group of 8 patients for which both echo data were available and at least $10 \mathrm{AF}$ episodes were recorded along with the ECG, being suitable for analysis. We found that both $\beta_{1}$ and $\mu$ are weakly correlated with atrial volume $(r=0.34$ and $r=0.19$, respectively), whereas $\mu$ is correlated with atrial strain ( $r=$ $-0.74, p<0.1)$ and AF burden $(r=0.68, p<0.05)$. Weak correlation between $\beta_{1}$ and AF burden was found $(r=0.29)$.

\section{Discussion and conclusion}

Identification of risk factors in paroxysmal AF patients has been limited to AF burden and a few other simple measures, such as minimum episode duration, but little attention has been paid up to now to the temporal distribution of AF episodes. The heterogeneity in AF progression is in agreement with the diversity of temporal AF episode patterns observed among patients. A model-based approach using the alternating bivariate Hawkes model has been recently proposed in an attempt to provide a more detailed characterization of episode patterns [5].

The model has been successfully applied to real data using long-term ECG recordings from patients with paroxysmal AF. In this study, model parameters, accounting for rhythm dominance, $\mu$, (AF or non-AF) and the degree of episode clustering, $\beta_{1}$, were estimated and their association with atrial volume and strain measurements investigated. Although the results of this study are not conclusive due to the small sample size, they still shed some light on this research line. Atrial structural remodeling is associated with changes in AF characteristics, often manifested as episodes of increasing duration (i.e., increasing 

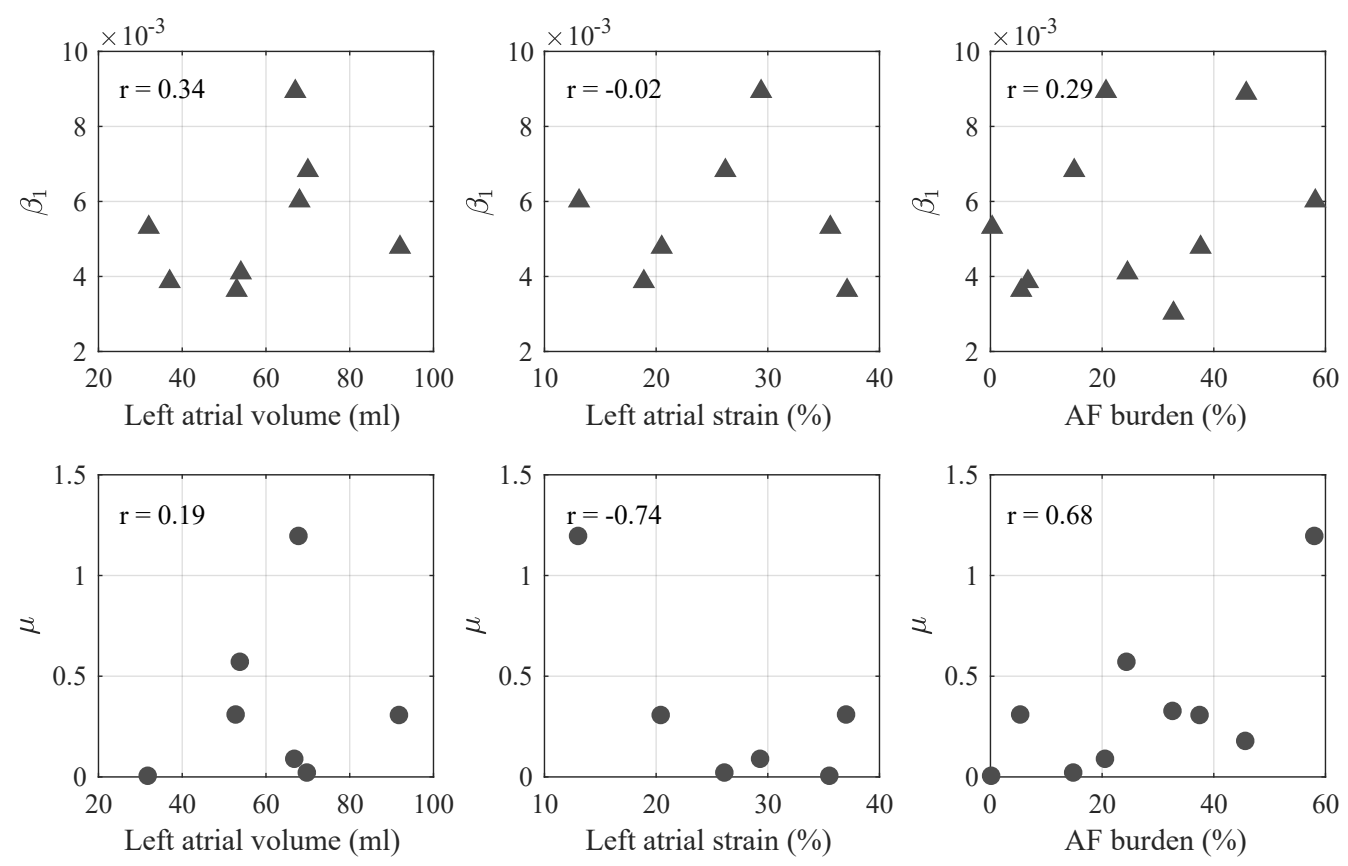

Figure 3. Association between model parameters, $\beta_{1}$ and $\mu$, and atrial volume, strain, and AF burden.

$\mu$ ). Therefore, $\mu$ may reflect the degree of atrial electrical and structural remodeling. Moreover, the low correlation between $\beta_{1}$ and $\mathrm{AF}$ burden suggests that cluster information provides information complementary to AF burden, which may be useful for risk assessment of ischemic stroke and as well as for better understanding of AF progression.

Nevertheless, further investigation in larger databases is still required in order to confirm the clinical value of the model parameters. A prospective study of the association between cluster information and stroke would help in the identification of potential risk factors in $\mathrm{AF}$, one of the main challenges that public health systems are facing today, due to the ageing population and the increasing prevalence of this arrhythmia.

\section{Acknowledgements}

This work was supported by the Swedish Research Council (grant 2016-03382) and European Regional Development Fund (grant 01.2.2-LMT-K-718-03-0027).

\section{References}

[1] R. Mahajan et al. "Subclinical device-detected atrial fibrillation and stroke risk: a systematic review and metaanalysis,” Eur. Heart J., vol. 39, pp. 1407-1415, 2018.

[2] G. Boriani et al. "AF burden is important-fact or fiction?," Int. J. Clin. Pract., vol. 68, pp. 444-452, 2014.

[3] A. S. Go et al. "Association of burden of atrial fibrillation with risk of ischemic stroke in adults with paroxysmal atrial fibrillation: The KP-RHYTHM study," JAMA Cardiol., vol. 3, pp. 601-608, 2018.

[4] L. Mont and E. Guasch, "Atrial fibrillation progression: How sick is the atrium?," Heart Rhythm, vol. 14, pp. 808809, 2017.

[5] M. Henriksson et al. "Modeling and estimation of temporal episode patterns in paroxysmal atrial fibrillation," IEEE Trans. Biomed. Eng., DOI: 10.1109/TBME.2020.2995563.

[6] A. G. Hawkes, "Spectra of some self-exciting and mutually exciting point processes," Biometrika, vol. 58, pp.83-90, 1971.

[7] J. G. Rasmussen, "Lecture Notes: Temporal point processes and the conditional intensity function.," arXiv: 1806.00221v1 [stat.ME], 2018.

[8] J. P. Martínez et al. "A wavelet-based ECG delineator: Evaluation on standard databases," IEEE Trans. Biomed. Eng., vol. 51, pp. 570-581, 2004.

[9] A. Petrenas et al. "Detection of occult paroxysmal atrial fibrillation," Med. Biol. Eng. Comput., vol. 53, pp. 287-297, 2015.

[10] A. Petrènas et al. "Low-complexity detection of atrial fibrillation in continuous long-term monitoring," Comput. Biol. Med., vol. 65, pp. 184-191, 2015.

[11] D. J. Daley and D. Vere-Jones, "An Introduction to the Theory of Point Processes: Volume I: Elementary Theory and Methods," 2nd ed., Springer, 2003.

Address for correspondence:

Alba Martín-Yebra, Department of Biomedical Engineering Lund University, Lund, Sweden

alba.martin@bme.lth.se 